\title{
Preparation of Gold Nanocomposites with Tunable Charge and Hydrophobicity via the Application of Polymer/Surfactant Complexation
}

\author{
Krisztina Bali, ${ }^{\dagger}$ György Sáfrán, ${ }^{\ddagger}$ Béla Pécz, ${ }^{\ddagger}$ and Róbert Mészáros ${ }^{*}, \dagger, \S$ \\ ${ }^{\dagger}$ Laboratory of Interfaces and Nanosized Systems, Institute of Chemistry, Eötvös Loránd University, Pázmány Péter sétány 1/A, \\ H-1117 Budapest, Hungary \\ ${ }^{\ddagger}$ Research Institute for Technical Physics and Materials Sciences (RITP), Centre for Energy Research, H.A.S., Konkoly Thege M. út \\ 29-33, H-1121 Budapest, Hungary \\ ${ }^{\S}$ Department of Chemistry, University J. Selyeho, 94501 Komárno, Slovakia
}

\section{Supporting Information}

\begin{abstract}
During the synthesis of gold nanoparticle (NP) assemblies, the interfacial charge and hydrophobicity of the primary particles play a distinguished role. In the present article, we demonstrate that the association of poly(ethyleneimine) (PEI) capped gold NPs with sodium alkyl sulfates provide a powerful route for the manipulation of these interfacial properties. Dynamic light-scattering, electrophoretic mobility, UV-vis-near-infrared spectroscopy, nanoparticle tracking analysis, and transmission electron microscopy measurements were used to characterize the PEI/surfactant/ gold nanoassemblies. The results indicate the formation of gold NPs surrounded by a PEI/surfactant shell with composition-dependent charge and hydrophobicity. The mean size and the aggregation of the nanoassemblies can be fine tuned by the amount of surfactant bound to the primary gold NPs as well as by the application of controlled mixing methods. The specific features of the prepared nanocomposites may be further exploited in next-generation applications.
\end{abstract}

\section{INTRODUCTION}

Gold nanoparticles (NPs) and their assemblies are promising candidates in a diversity of fields, including biomedical ${ }^{1-4}$ or photochemical and optical devices. ${ }^{5-8}$ In nearly all of these applications, the surface charge and hydrophobicity of the NPs must be strictly controlled and adjusted. For instance, the surface charge of gold NPs can seriously affect their cellular uptake and biodistribution. ${ }^{9,10}$ Furthermore, the hydrophobic nature of their surface layer may considerably affect the charge transfer of the individual NPs. ${ }^{11}$ In addition, both parameters crucially affect the interparticle interactions as well as the formation of selforganized or directed gold assemblies. ${ }^{12-14}$

Various macromolecules bound onto gold NPs are good candidates for adjusting charge and hydrophobicity through their functionalization. ${ }^{15}$ These polymers also provide (electro)steric stabilization after the nucleation of gold NPs. Recent studies have revealed that several polymers, such as Pluronic triblock copolymers ${ }^{16,17}$ or polyamines, ${ }^{18,19}$ play specific multiple roles in the synthesis of gold NPs, i.e., they could simultaneously act as reducing and stabilizing agents. ${ }^{16-19}$ Thus, stable dispersions of macromolecule-entrapped noble-metal NPs can be prepared without the need of reducing compounds.
Branched poly(ethyleneimines) (PEIs), in particular, provide an economic alternative for the synthesis of dendrimer capped gold NPs due their low cost and commercial availability. ${ }^{20-23}$ In this case, a mild reduction of gold(III) ions occurs with a pronounced role of the secondary amine groups. The reduction and nucleation processes result in either single $\mathrm{NPs}^{20,21}$ or supraparticles embedded in the PEI matrix. ${ }^{22,23}$ In addition, functionalized branched PEI molecules have also been applied recently for the synthesis of gold NPs with promising properties. PEGylated PEI-entrapped gold NPs, for instance, were utilized for computed tomography imaging and polymerase chain reactions. $^{24,25}$ It was also shown that the functionalization of amine groups with alkyl chains or maltose units could significantly affect the morphology of gold NPs, including the appearance of anisometric nanohybrids. ${ }^{26,27}$

In principle, an alternative of surface functionalization may be provided using the association of surfactants with polymerentrapped noble-metal nanoparticles. Earlier studies revealed that the nonequilibrium polyelectrolyte (PE)/surfactant (S)

Received: October 23, 2017

Accepted: November 17, 2017

Published: December 7, 2017 

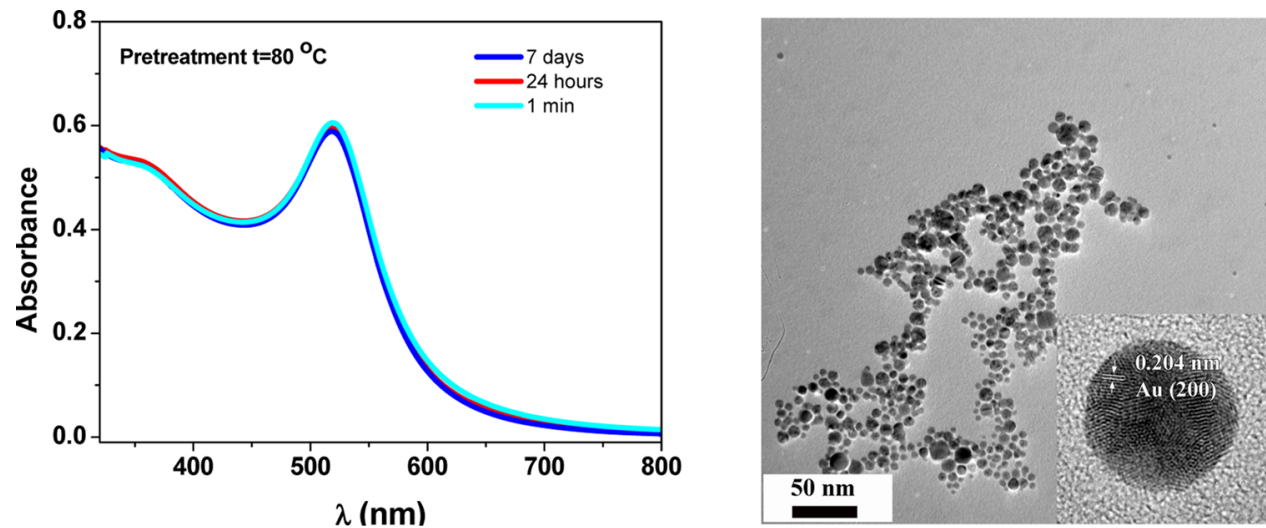

Figure 1. UV-vis spectra of PEI-Au NPs in the $\mathrm{HAuCl}_{4}+\mathrm{PEI}$ system at $\mathrm{pH}=4$ after $2 \mathrm{~h}$ pretreatment at $80{ }^{\circ} \mathrm{C}$, followed by measurements at $25^{\circ} \mathrm{C}$ (left). TEM pictures of the prepared gold NPs (right). It should be noted that the agglomeration of individual positively charged NPs shown in the picture is not the consequence of bulk assembly formation but due to their aggregation on the negatively charged carbon grid during TEM sample preparation.

association can be tuned to prepare PE/S NPs with controlled size, structure, and chemistry via the adequate choice of the oppositely charged components as well as of the co-associating materials. ${ }^{28-35}$ Specifically, it was shown that through the application of well-defined preparation methods, stable polyamine/anionic surfactant nanophases ${ }^{28-30}$ or nanolayers ${ }^{36,37}$ of variable size, charge, structure, and hydrophobicity can be produced.

In the present article, the association of PEI-entrapped gold NPs with sodium alkyl sulfates will be explored using dynamic light-scattering (DLS), nanoparticle tracking analysis (NTA), electrophoretic mobility, UV-vis-near-infrared (NIR) spectroscopy, and transmission electron microscopy (TEM) techniques. To the best of our knowledge, this is the first systematic study of the direct application of PE/S complexation for the preparation of noble-metal nanohybrids with tunable properties. We will demonstrate that via the controlled addition and mixing with anionic surfactants, the charge and hydrophobicity of the PEI-capped gold NPs and their interparticle interactions can be adequately adjusted, resulting in the formation of bulk and solid gold nanocomposites.

\section{RESULTS AND DISCUSSION}

PEI-Capped Gold NPs. Earlier studies revealed the formation of quasi-spherical gold NPs as a result of the mild reduction and stabilizing effects of hyperbranched PEI molecules. However, the reported size distributions are controversial with respect to the role of absolute concentrations and ratio of $\mathrm{Au}(\mathrm{III})$ ions and ethyleneimine (EI) monomers. $^{20-23}$ In this work, the final concentrations of these components are fixed at $1.2 \mathrm{mM} \mathrm{EI}$ and $0.2 \mathrm{mM} \mathrm{HAuCl}_{4}$, in agreement with refs 18 and 20, to ensure optimal conditions for the detection of the nucleation of gold NPs but to avoid the large excess of free PEI molecules. The variation in the UV-vis spectra of the PEI-Au systems with time in Figure S1 clearly indicates that the reduction of gold(III) ions is a slow process at room temperature, in agreement with earlier studies. However, a much faster and reproducible way of PEI-capped gold NP synthesis was achieved after $2 \mathrm{~h}$ of incubation at $80^{\circ} \mathrm{C}$.

As shown in Figure 1 (left), the spectrum of the pretreated gold NP dispersions becomes time independent after the thermal pretreatment, and its shape is consistent with the formation of quasi-spherical particles. The polydispersity of the formed NPs is significant, and their mean diameters were found to be $20 \pm 2 \mathrm{~nm}$ by DLS and $12 \pm 4 \mathrm{~nm}$ by TEM (Figure 1 (right)). The observed localized surface plasmon resonance (LSPR) band maximum at $\lambda$ $=519 \mathrm{~nm}$ is in reasonable agreement with the diameter values predicted by the Mie theory or observed in aqueous medium. ${ }^{38}$

PEI/Surfactant/Gold Nanoassemblies. The complexation of PEI-Au NPs with anionic surfactants was investigated for mixtures, which were prepared through the application of the socalled rapid- and slow-mixing procedures ${ }^{33,34}$ developed earlier for the controlled preparation of oppositely charged PE/S systems. The photos in Figure 2a reveal a remarkable difference in some of the samples prepared via the two preparation methods. During the application of rapid-mixing procedure, precipitates are formed at intermediate STDS concentrations, whereas transparent (rosy) gold nanosystems are observable at larger or smaller surfactant concentrations. In the case of slowmixing procedure, however, opalescent systems are formed over a wide composition range (i.e., above a certain sodium tetradecyl sulfate (STDS) concentration, $\cong 0.3 \mathrm{mM}$ ) indicating the formation of large aggregates. Similar effect of the mixing methods was also observed for $\mathrm{PEI}-\mathrm{Au} /$ sodium dodecyl sulfate (SDS) systems.

In the light of these findings, the rapid-mixing protocol was utilized primarily to synthesize gold nanohybrids in the following part of our work. The association between the PEI-entrapped gold NPs and the alkyl sulfates is completed within a couple of hours, as demonstrated by the absorbance (at $\lambda=524 \mathrm{~nm}$ ) vs surfactant concentration curves in Figure $2 b, c$, except the intermediate surfactant concentration range, where slow aggregation of the NPs occurs.

Characteristic examples for the time dependence of the whole UV-vis spectra of PEI-Au/STDS and PEI-Au/SDS systems are shown in Figure S2a,b, respectively.

The mean mobility and the apparent mean hydrodynamic diameter of the bulk nanocomposites against the surfactant concentration is plotted in Figure $3 a, b$, for STDS and SDS containing nanoassemblies, respectively, prepared by rapidmixing method. Several examples for the monomodal size distributions of the nanoassemblies, determined by DLS, are shown in Figure S3.

The net positive charge of the nanohybrids decreases, whereas their size increases with increasing surfactant concentration up to the appearance of precipitation. The charge neutralization surfactant concentration is smaller for STDS $(0.3 \mathrm{mM})$ than for SDS $(0.45 \mathrm{mM})$ due to the higher driving force of STDS 
(a)

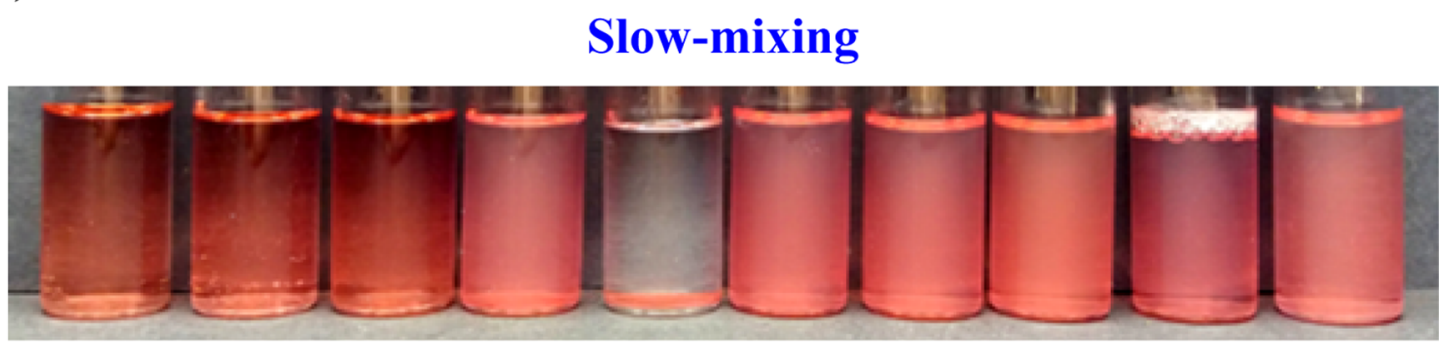

\section{Rapid-mixing}

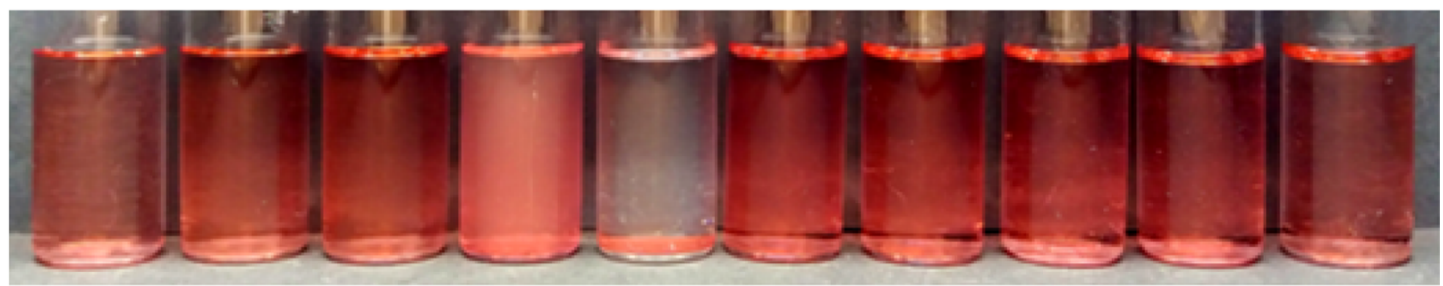

\section{$c_{\text {STDS }}$}

(b)

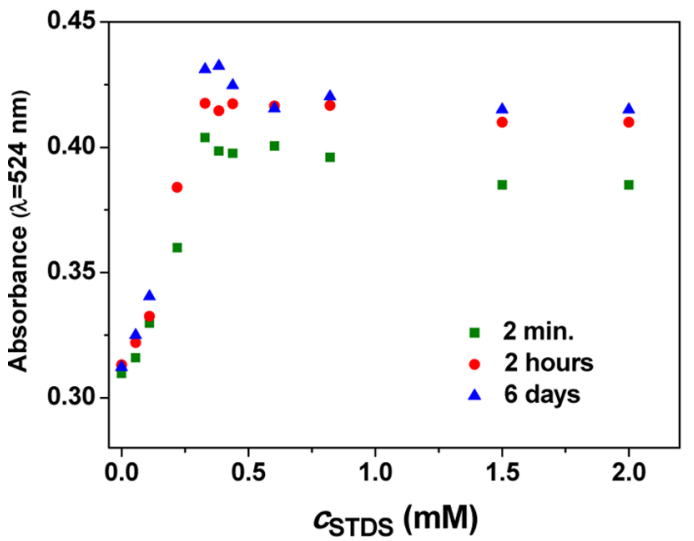

(c)

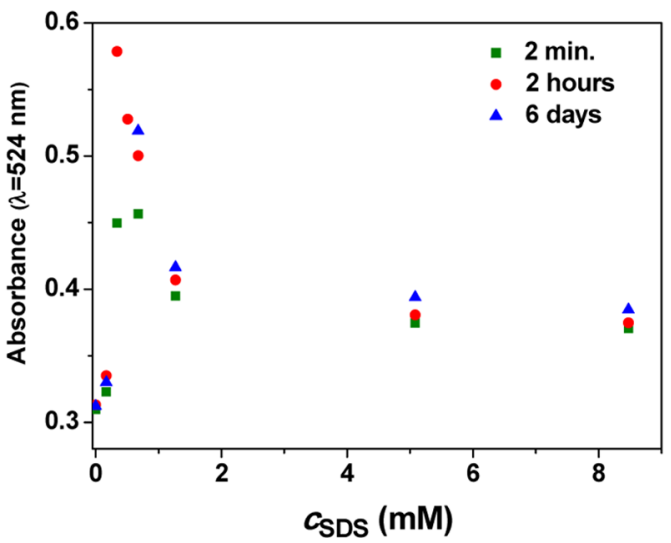

Figure 2. (a) Photos of PEI-Au/STDS mixtures prepared by the slow- (top) and rapid-mixing (bottom) methods. The concentration of STDS increases from left to right. Time dependence of the absorbance (at $\lambda=524 \mathrm{~nm}$ ) vs surfactant concentration curves of (b) PEI-Au/STDS and (c) PEI$\mathrm{Au} / \mathrm{SDS}$ gold nanosystems made by the rapid-mixing protocol. All of the mixtures were prepared from the PEI-Au NPs, synthesized by the $2 \mathrm{~h}$ of thermal pretreatment at $80^{\circ} \mathrm{C}$.

binding on the PEI molecules. A further increase in the surfactant concentration leads to charge reversal and decrease in the size of the nanoassemblies, which levels off in the vicinity of the bulk critical micelle concentration (cmc) of the amphiphiles. As indicated inFigure S4, the mean size of the bulk gold nanoassemblies is not dependent on the time within the experimental error over a wide concentration range, as checked by DLS for 2 weeks (except in the vicinity of charge neutralization where slow aggregation is observable).

At two selected initial compositions, the mean Stokes diameters were also determined from NTA measurements in the concentration range of surfactant excess. As shown in Figure $3 \mathrm{a}, \mathrm{b}$, the $d_{\mathrm{H}}$ values from NTA to DLS are in good agreement. Furthermore, the three-dimensional representation of the particle concentration vs scattered intensity and Stokes diameter functions (Figures S6 and S7) unambiguously reveals the existence of only one population of gold nanoassemblies (i.e., the negligible amount of free PEI/surfactant complexes not bound to the primary gold NPs).

The variation in the mean size of the gold NPs with sodium alkyl sulfate concentration correlates qualitatively with the observed small blue shift at low and the slight red shift at high surfactant concentrations, as shown by the UV-vis spectra in Figure $3 \mathrm{c}$,d, respectively. The lack of additional absorbance peaks in the near IR regime (see also Figure S5 in the SI) is consistent with the quasi-spherical shape of the particles. The enhanced absorbance compared to the bare gold NPs is mainly attributable to the aggregation of the primary gold NPs coated with PEI/ surfactant layers into larger assemblies with positive or negative net charge (an example is given in Figure 3e). On the other hand, the significantly reduced absorbance and the slight red shift of the LSPR band maximum at the bulk cmc of SDS and STDS ( $521 \mathrm{~nm}$ compared to $519 \mathrm{~nm}$ of the PEI-capped gold NPs) suggest the formation of individual, overcharged gold NPs at these compositions. This is also consistent with the TEM picture in 
(a)

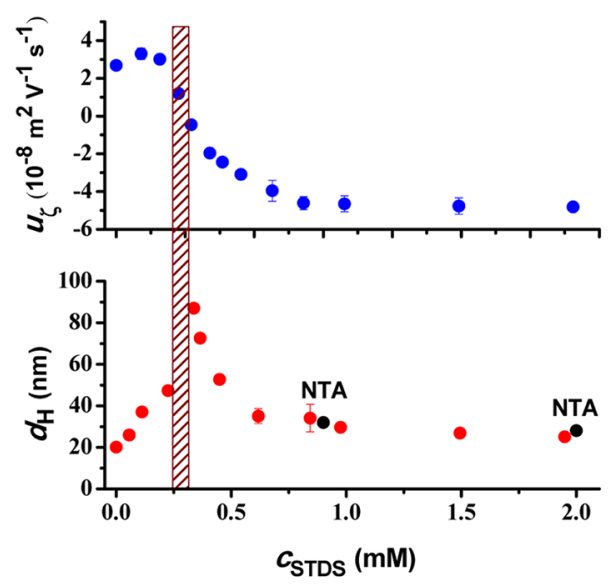

(c)

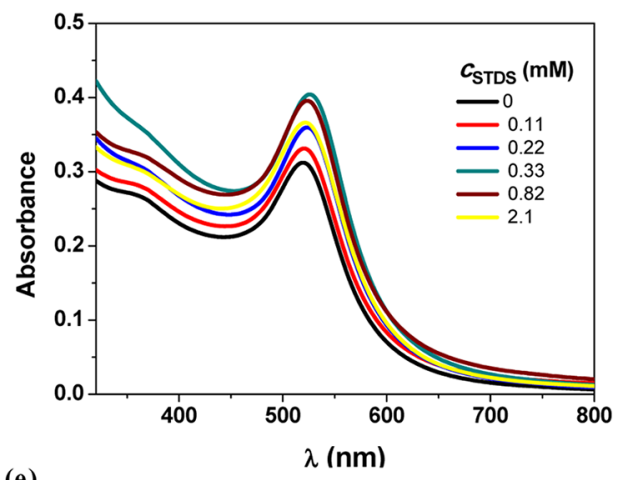

(e)

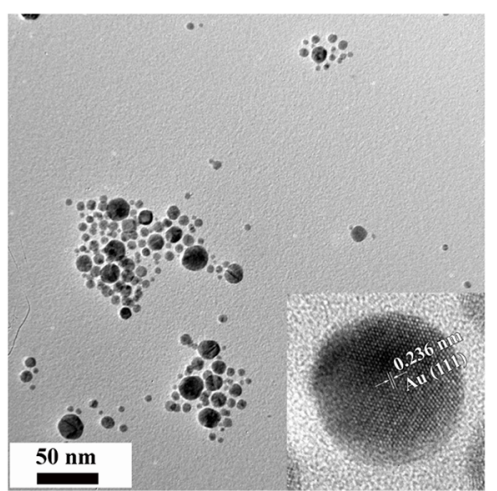

(b)

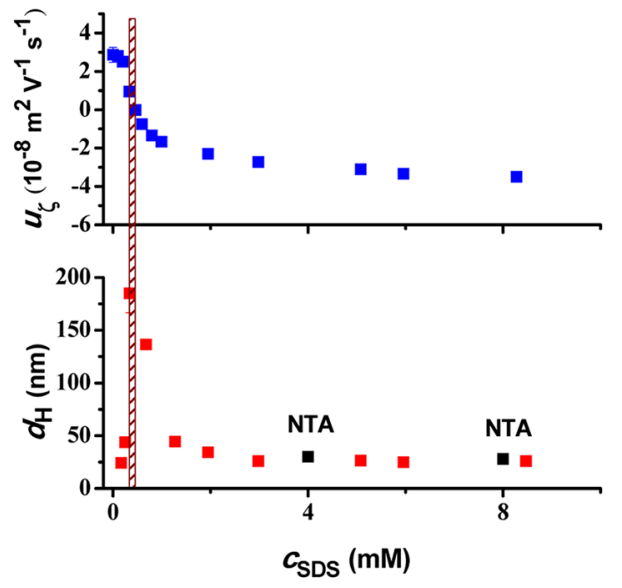

(d)

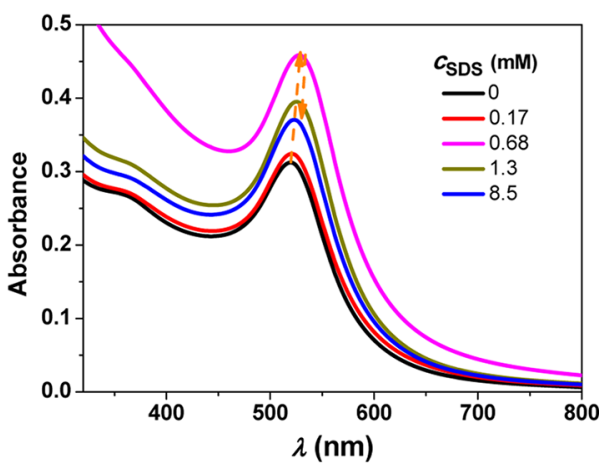

(f)

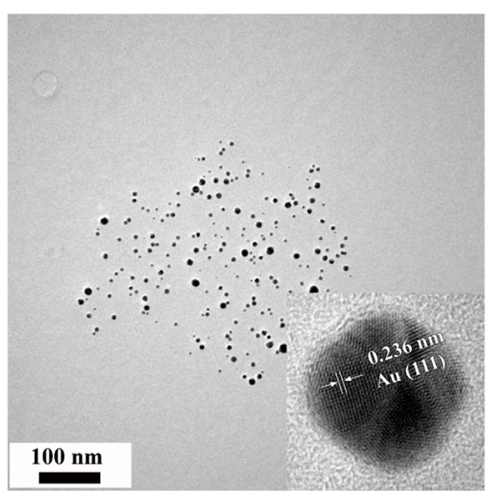

Figure 3. $(\mathrm{a}, \mathrm{b})$ Variation in the mean electrophoretic mobility $\left(u_{\epsilon}\right)$ and the apparent mean diameter $\left(d_{\mathrm{H}}\right)$ with the surfactant concentration of PEI-Au/ STDS and PEI-Au/SDS nanoassemblies, respectively, prepared through the rapid-mixing protocol. The $d_{\mathrm{H}}$ values were determined by the DLS (red symbols) and the NTA techniques (black symbols) as well. (c, d) Surfactant concentration dependence of the UV-vis spectra of the same systems as in (a) and (b). (e, f) TEM pictures of PEI-Au/STDS nanocomposites, prepared via rapid-mixing method at 0.41 and 2.0 mM STDS concentrations, respectively.

Figure $3 \mathrm{f}$ as well as with the similar Stokes diameters measured at the $\mathrm{cmc}(25 \pm 2 \mathrm{~nm})$ and in the absence of surfactant $(20 \pm 2$ $\mathrm{nm})$.

Figure 4 shows the comparison of the mean Stokes diameters and UV-vis spectra of the PEI-Au/STDS nanocomposite dispersions made by the two types of mixing protocols at the same compositions. The graphs clearly reveal that, in contrast to rapid-mixing methods, the major consequence of the application of slow-mixing method is manifested in the formation of large aggregates of gold NPs over a wide concentration range of surfactant excess, in agreement with Figure S8 and the visual appearance of the samples in Figure 2a as well. However, the observed spectra and the measured $d_{\mathrm{H}}$ values did not reveal any time dependence for 2 weeks within the experimental error.

The interpretation of our observations is summarized in Figure 5. The PEI-capped gold NPs are positively charged due to the excess protonated amine groups of the polymer. We speculate that essentially the charged primary amine groups provide the electrostatic stabilization of the hyperbranched PEI-entrapped gold NPs because the secondary amine groups provide the strongest reduction capability. ${ }^{39}$ This is also supported qualitatively by the amounts of surfactant bound onto the PEIcapped NPs, which can be estimated as 0.2 and $0.35 \mathrm{mM}$ for SDS and STDS, respectively, at zero mobility of the gold nanohybrids 

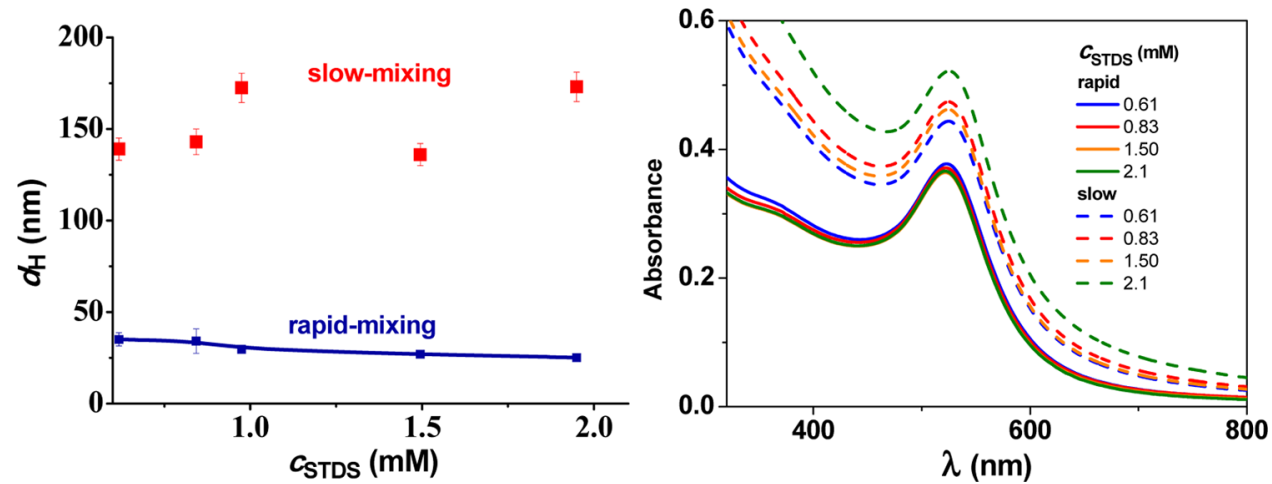

Figure 4. Comparison of the apparent mean diameter vs surfactant concentration curves (left) and the UV-vis spectra (right) of PEI-Au/STDS mixtures prepared via the slow- and rapid-mixing protocols, respectively, at surfactant excess.

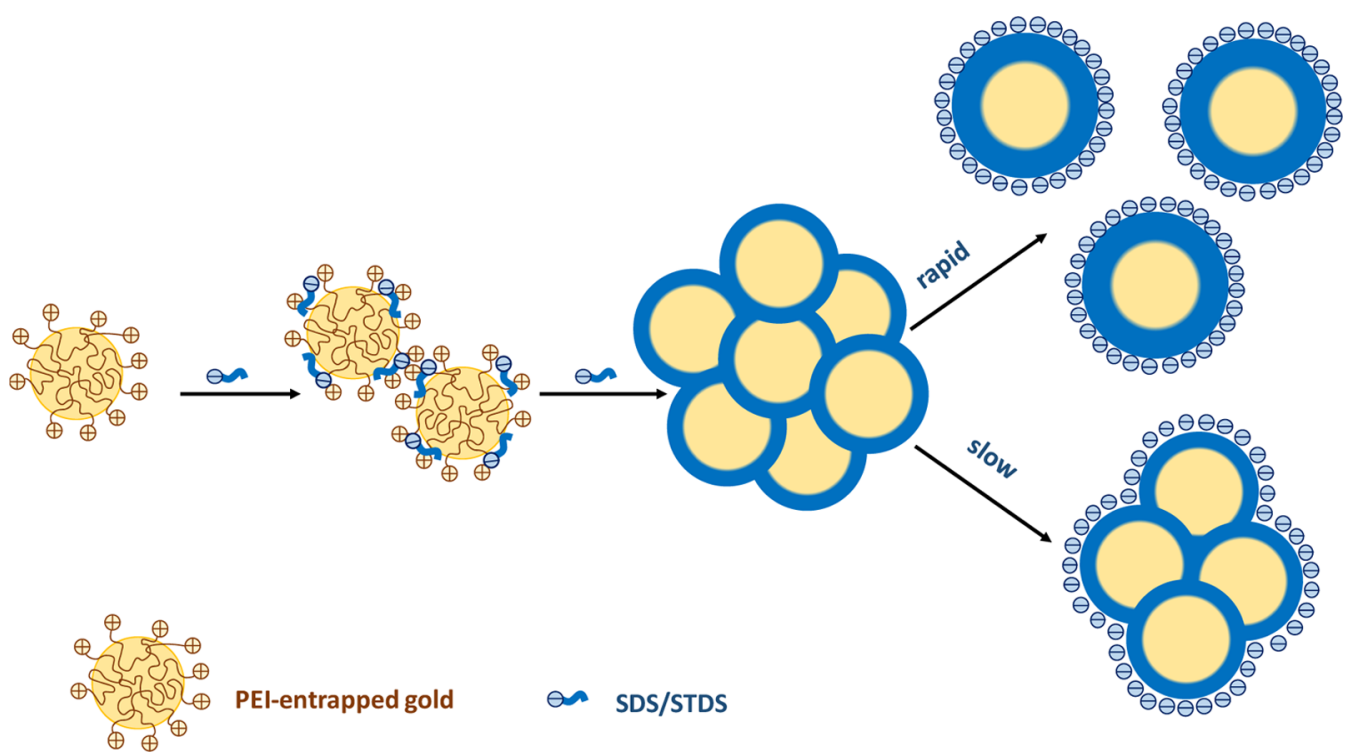

Figure 5. Schematic representation of the assembly formation of PEI-capped gold NPs due to their interaction with anionic surfactant molecules upon application of the rapid- and slow-mixing protocols.

(assuming similarly low $(\leq 0.1 \mathrm{mM})$ equilibrium free surfactant concentration under these conditions as in the case of bulk PEI/ SDS complexes with $\left.u_{\varsigma}=0\right){ }^{40}$ These values are roughly commensurable with the primary amine group concentration of PEI.

With increasing surfactant concentration, the binding of the alkyl sulfate ions to the amine groups compensate the positive net charge of the PEI-Au NPs, leading to decrease in charge and increase in hydrophobicity of their external layer (similarly to the formation of compact hydrophobic PEI/SDS layers on silica or mica $\left.^{36,37}\right)$. This leads to an increase in the mean size of the nanocomposites due to the aggregation of the gold NPs surrounded by the hydrophobic polyelectrolyte/surfactant layers, and then solid precipitates are observable at intermediate surfactant concentrations. At even higher surfactant concentrations, the alkyl sulfate ions may bind onto the neutralized hydrophobic PEI/alkyl sulfate layer of the gold NPs similarly to the PEI/SDS-coated silica or mica surfaces in refs 36 and 37.

This adsorption process results in the charge reversal of the gold nanoassemblies and could prevent their coagulation at larger STDS or SDS concentrations, provided that the rapidmixing protocol was used for the preparation of the nanocomposites.
This phenomenon is analogous with the electrostatic stabilizing mechanisms of hydrophobic PEI/SDS NP dispersions made by the rapid-mixing procedure at surfactant excess and the observed impact of solution preparation methods on these systems. ${ }^{30,34,35}$ During the rapid-mixing process, the equilibrium free surfactant concentration and thus the corresponding excess charge of the NPs-due to the adsorbed surfactant ions-are achieved relatively quickly at high surfactant concentrations. Therefore, the overcharged gold nanoassemblies are not destabilized as a result of the initial concentration gradients, but remain stable, as demonstrated by the scheme in Figure 5 and in the TEM photograph in Figure 3f. In contrast, during slowmixing method, the surfactant is gradually dripped into the PEIcapped gold NP dispersion. This prevents the completion of the surfactant ion adsorption on the surface of the neutralized NPs before their irreversible coagulation into larger agglomerates occurs.

\section{CONCLUSIONS}

In conclusion, we have shown that branched PEI molecules can not only be used for the entrapment and stabilization of gold NPs, but their association with anionic surfactants can be utilized in the synthesis of nanohybrids with polyamine/surfactant shell around the gold core. The charge and hydrophobicity of this 
layer as well as the interparticle interactions of the NPs can be adequately adjusted through the variation in polymer/surfactant ratios, provided that the well-defined rapid-mixing method is used. It was demonstrated that only this preparation method provides the stable dispersion of small, negatively charged gold nanohybrids with reproducible mean size. These findings may be further utilized for the controlled synthesis of aqueous dispersions and solid assemblies of noble-metal NPs with hierarchical structure and tunable properties. Finally, the synthesis of biosurfactant/polymer-coated NPs in the future may lead to novel nanocomposites with potential biomedical applications.

\section{METHODS}

Materials. The sodium dodecyl sulfate and sodium tetradecyl sulfate (SDS and STDS, Sigma-Aldrich, $\geq 98.0 \%$ ) samples were recrystallized twice from ethanol. The cmc of SDS and STDS in water was found to be 8.1 and $1.9 \mathrm{mM}$, respectively, at $25.0 \pm 0.1$ ${ }^{\circ} \mathrm{C}$ from conductivity measurements. The branched poly(ethyleneimine) (PEI, Sigma-Aldrich) sample had a mass averaged molar mass of $25 \mathrm{kDa}$, with an approximate 1:2:1 ratio of the primary, secondary, and tertiary amine groups. ${ }^{28}$ Ultrapure water (Milli-Q) was used for the preparation of the solutions.

Solution Preparation Methods. The aqueous solutions of $\mathrm{HAuCl}_{4}$ was added to poly(ethyleneimine) (PEI) solutions to prepare a $20 \mathrm{~mL}$ mixture under continuous stirring with a magnetic stirrer $(1800 \mathrm{rpm})$. The final ethyleneimine (EI) monomer and $\mathrm{HAuCl}_{4}$ concentrations were 1.2 and $0.2 \mathrm{mM}$, respectively, resulting in a mixture with $\mathrm{pH} \approx 4$. In the preliminary experiments, these mixtures were stored at room temperature and analyzed by UV-vis spectroscopy for a week. However, to achieve reproducible results and faster equilibration with the added surfactants, in the majority of the experiments, the mixtures were pretreated at $80^{\circ} \mathrm{C}$ for $2 \mathrm{~h}$ (immediately after their preparation at room temperature). The as-prepared gold colloids were stored at $t=25.0 \pm 0.5^{\circ} \mathrm{C}$ and used for further experiments within 1 day. For the synthesis of $\mathrm{PEI}-\mathrm{Au} /$ surfactant nanocomposites, two types of mixing methods, developed earlier for $\mathrm{PE} / \mathrm{S}$ systems, ${ }^{33,34}$ were used. During the so-called rapidmixing ${ }^{33,34}$ method, equal volumes $(1.5 \mathrm{~mL})$ of SDS or STDS solution (at double their intended final concentration) and PEIcapped gold NP dispersion were quickly mixed under continuous stirring with a magnetic stirrer $(1800 \mathrm{rpm})$. In the case of slowmixing method, ${ }^{34,35}$ the surfactant solution was added slowly, drop-by-drop, to the PEI-Au dispersion of equal volume (1.5 $\mathrm{mL}$ ) under continuous stirring with a magnetic stirrer. All of the mixtures were made and stored at $t=25.0 \pm 0.5{ }^{\circ} \mathrm{C}$.

UV-Vis-NIR Spectroscopy. The formation of gold nanoparticles was monitored by observing changes in the absorption spectra at $25{ }^{\circ} \mathrm{C}$. A Perkin-Elmer Lambda 1050 $\mathrm{UV} / \mathrm{vis} / \mathrm{NIR}$ spectrophotometer was used to record the spectra in the 320-1000 nm wavelength interval with a path length of $1.00 \mathrm{~cm}$. Because the detected absorbance values were negligible in the near-IR region (see Figure S5 of the SI), the major part of the measurements was carried out only in the UV-vis range of the spectra.

Electrophoretic Mobility Measurements. The mean electrophoretic mobility $\left(u_{\zeta}\right)$ of the gold/polymer/surfactant nanocomposites was determined at $25.0 \pm 0.1{ }^{\circ} \mathrm{C}$, immediately after solution preparation, using a Malvern Zetasizer Nano ZSP instrument. The apparatus utilizes the M3-PALS technique to determine the mean velocity of the $\mathrm{PE} / \mathrm{S}$ complexes $\left(v_{\mathrm{E}}\right)$ at a given electric field strength $(E)$, from the measured frequency shift of the scattered light due to the movement of the particles. The mean mobility values are derived from the $u_{\zeta}=v_{\mathrm{E}} / E$ relationship. In the case of the transparent systems, the measurements were repeated after $24 \mathrm{~h}$, but no deviations from the previously measured data were found within the experimental error.

Dynamic Light-Scattering (DLS) Measurements. The mean hydrodynamic size of the polyelectrolyte/surfactant complexes was determined by dynamic light-scattering (DLS). The experimental setup (Brookhaven Instruments) consisted of a BI-200SM goniometer system and a BI-9000 AT digital correlator using a Genesis MX488-1000 OPS laser (1 W). The measurements were carried out at $\lambda=488 \mathrm{~nm}$ wavelength, $\theta=$ $90^{\circ}$ scattering angle, and at $25.0 \pm 0.1{ }^{\circ} \mathrm{C}$ for $24 \mathrm{~h}$, as well as 2 weeks after the preparation of the systems. The CONTIN analysis of the normalized electric field autocorrelation functions revealed wide unimodal distributions of the nanocomposites in the investigated composition range. The apparent mean diffusion coefficient of the particles $\left(D_{\text {app }}\right)$ was derived from the secondorder cumulant analysis of the autocorrelation function and their apparent mean hydrodynamic diameter $\left(d_{\mathrm{H}}\right)$ was calculated on the basis of the Einstein-Stokes relation: $D_{\text {app }}=\frac{k_{\mathrm{B}} T}{3 \pi \eta d_{\mathrm{H}}}$, where $T$ is the temperature, $k$ is the Boltzmann constant, $\eta$ is the viscosity of the medium, and $d_{\mathrm{H}}$ is the diameter of the particle. Occasionally, the Stokes diameters were also determined from the DLS measurements carried out at $\theta=175^{\circ}$ scattering angle by the backscattering utility of the previously described Malvern Zetasizer Nano ZSP instrument using a $10 \mathrm{~mW} \mathrm{He}-\mathrm{Ne}$ laser at $\lambda$ $=633 \mathrm{~nm}$. In the investigated transparent concentration range, the observed $d_{\mathrm{H}}$ values were not dependent on the type of the applied DLS setups. The turbid concentration range was only monitored by the backscattering method because this technique considerably suppresses the effect of multiple scattering.

Nanoparticle Tracking Analysis (NTA). For the determination of the mean size of the nanocomposites, the NTA technology (Nanosight LM10) was also applied. Prior to injecting the gold NP dispersions, the cell was cleaned by isopropyl alcohol and dried by high-purity nitrogen jet. A laser beam ( $40 \mathrm{~mW}$, at $\lambda=642 \mathrm{~nm}$ ) was passed through the sample chamber and the motion of the particles in the path of this beam was visualized using a microscope with a charge-coupled device camera, operating at 30 frames/s. The videos were analyzed using the NTA 3.1 software and the apparent diffusion coefficient of the individual particles was calculated from their mean square displacement, $\overline{(x, y)}^{2}: D_{\text {app }}=\frac{\overline{(x, y)}^{2}}{4 t}$, where $x$ and $y$ are the twodimensional coordinates and $t$ is the time of particle tracking. The apparent diameter of the individual particles was calculated according to the Einstein-Stokes equation. A unique feature of the NTA technique is that the scattered intensity and the size of the particles can be determined independently, and thus nanoparticles with largely different refractive indices could be distinguished. It should be noted, however, that no information about the shape and structure of the particles can be resolved by the NTA method in the investigated nanoscale range. In addition, accurate tracking of the particles is only possible for very dilute nanosystems with typical concentrations between $10^{6}$ and $10^{10}$ particles $/ \mathrm{cm}^{3}$. Therefore, the gold nanocomposite dispersion was diluted 100 -fold with polymer free surfactant solution of the same analytical SDS or STDS concentration than 
that of the dispersion. These diluted systems were filtered through $0.22 \mu \mathrm{m}$ membrane filters before the measurements.

Transmission Electron Microscopy (TEM). The diluted NPs were drop-dried on carbon-coated microgrids for the TEM study. A Philips CM $20(200 \mathrm{kV})$ microscope was used for the conventional electron microscopy (bright field and dark field images), whereas a JEOL 3010 (300 kV, point resolution: 0.17 $\mathrm{nm}$ ) equipped with Gatan Tridiem electron energy loss spectroscopy was used for high-resolution TEM investigations.

\section{ASSOCIATED CONTENT}

\section{S Supporting Information}

The Supporting Information is available free of charge on the ACS Publications website at DOI: 10.1021/acsomega.7b01623.

Additional graphs related to the formation and characterization of PEI-Au NPs and PEI-Au/surfactant nanocomposites (PDF)

\section{AUTHOR INFORMATION}

\section{Corresponding Author}

*E-mail: meszaros@chem.elte.hu. Phone: +36-1-372-2500/ 1906.

ORCID $\odot$

Róbert Mészáros: 0000-0001-9638-2207

Notes

The authors declare no competing financial interest.

\section{ACKNOWLEDGMENTS}

This publication is the partial result of the Research \& Development Operational Programme for the project "Modernization and Improvement of Technical Infrastructure for Research and Development of J. Selye University in the Fields of Nanotechnology and Intelligent Space", ITMS 26210120042, co-funded by the European Regional Development Fund. This work was also supported by the Hungarian Scientific Research Fund (OTKA K 108646), which is gratefully acknowledged.

\section{REFERENCES}

(1) Dreaden, E. C.; Alkilany, A. M.; Huang, X.; Murphy, C. J.; El-Sayed, M. A. The Golden Age: Gold Nanoparticles for Biomedicine. Chem. Soc. Rev. 2012, 41, 2740-2779.

(2) Abadeer, N. S.; Murphy, C. J. Recent Progress in Cancer Thermal Therapy Using Gold Nanoparticles. J. Phys. Chem. C 2016, 120, 46914716.

(3) Yeh, Y.-C.; Crerana, B.; Rotello, V. M. Gold Nanoparticles: Preparation, Properties, and Applications in Bionanotechnology. Nanoscale 2012, 4, 1871-1880.

(4) Li, J.; Liu, J.; Chen, C. Remote Control and Modulation of Cellular Events by Plasmonic Gold Nanoparticles: Implications and Opportunities for Biomedical Applications. ACS Nano 2017, 11, 2403-2409.

(5) Schlather, A. E.; Manjavacas, A.; Lauchner, A.; Marangoni, V. S.; DeSantis, C. J.; Nordlander, P.; Halas, N. J. Hot Hole Photoelectrochemistry on Au@SiO2@Au Nanoparticles.J.Phys. Chem. Lett. 2017, 8, 2060-2067.

(6) Paterson, S.; Thompson, S. A.; Gracie, J.; Wark, A. W.; de la Rica, R. Self-Assembly of Gold Supraparticles with Crystallographically Aligned and Strongly Coupled Nanoparticle Building Blocks for SERS and Photothermal Therapy. Chem. Sci. 2016, 7, 6232-6237.

(7) Yang, G.; Hu, L.; Keiper, T. D.; Xiong, P.; Hallinan, D. T., Jr. Gold Nanoparticle Monolayers with Tunable Optical and Electrical Properties. Langmuir 2016, 32, 4022-4033.

(8) Yang, G.; Nanda, J.; Wang, B.; Chen, G.; Hallinan, D. T., Jr. SelfAssembly of Large Gold Nanoparticles for Surface-Enhanced Raman Spectroscopy. ACS Appl. Mater. Interfaces 2017, 9, 13457-13470.
(9) Jiang, Y.; Huo, S.; Mizuhara, T.; Das, R.; Lee, Y.; Hou, S.; Moyano, D. F.; Duncan, B.; Liang, X.; Rotello, V. M. The Interplay of Size and Surface Functionality on the Cellular Uptake of Sub-10 nm Gold Nanoparticles. ACS Nano 2015, 9, 9986-9993.

(10) Elci, S. G.; Jiang, Y.; Yan, B.; Kim, S. T.; Saha, K.; Moyano, D. F.; Tonga, G. Y.; Jackson, L. C.; Rotello, V. M.; Vachet, R. W. Surface Charge Controls the Suborgan Biodistributions of Gold Nanoparticles. ACS Nano 2016, 10, 5536-5542.

(11) Goldmann, C.; Lazzari, R.; Paquez, X.; Boissiére, C.; Ribot, F.; Sanchez, C.; Chanéac, C.; Portehault, D. Charge Transfer at Hybrid Interfaces: Plasmonics of Aromatic Thiol-Capped Gold Nanoparticles. ACS Nano 2015, 9, 7572-7582.

(12) Stolarczyk, J. K.; Deák, A.; Brougham, D. F. Nanoparticle Clusters: Assembly and Control Over Internal Order, Current Capabilities, and Future Potential. Adv. Mater. 2016, 28, 5400-5424.

(13) Sánchez-Iglesias, A.; Grzelczak, M.; Altantzis, T.; Goris, B.; PérezJuste, J.; Bals, S.; Van Tendeloo, G.; Donaldson, S. H., Jr.; Chmelka, B. F.; Israelachvili, J. N.; Liz-Marzán, L. M. Hydrophobic Interactions Modulate Self-Assembly of Nanoparticles. ACS Nano 2012, 6, 1105911065 .

(14) Grzelczak, M.; Liz-Marzán, L. M. Exploiting Hydrophobic Interactions at the Nanoscale. J. Phys. Chem. Lett. 2014, 5, 2455-2463.

(15) Daniel, M.-C.; Astruc, D. Gold Nanoparticles: Assembly, Supramolecular Chemistry, Quantum-Size-Related Properties, and Applications toward Biology, Catalysis, and Nanotechnology. Chem. Rev. 2004, 104, 293-346.

(16) Sakai, T.; Alexandridis, P. Single-Step Synthesis and Stabilization of Metal Nanoparticles in Aqueous Pluronic Block Copolymer Solutions at Ambient Temperature. Langmuir 2004, 20, 8426-8430.

(17) Khullar, P.; Singh, V.; Mahal, A.; Kaur, H.; Singh, V.; Banipal, T. S.; Kaur, G.; Bakshi, M. S. Tuning the Shape and Size of Gold Nanoparticles with Triblock Polymer Micelle Structure Transitions and Environments. J. Phys. Chem. C 2011, 115, 10442-10454.

(18) Sun, X.; Dong, S.; Wang, E. One-Step Synthesis and Characterization of Polyelectrolyte-Protected Gold Nanoparticles through a Thermal Process. Polymer 2004, 45, 2181-2184.

(19) Sun, X.; Dong, S.; Wang, E. High-Yield Synthesis of Large SingleCrystalline Gold Nanoplates through a Polyamine Process. Langmuir 2005, 21, 4710-4712.

(20) Sun, X.; Dong, S.; Wang, E. One-Step Preparation of Highly Concentrated Well-Stable Gold Colloids by Direct Mix of Polyelectrolyte and $\mathrm{HAuCl}_{4}$ Aqueous Solutions at Room Temperature. J. Colloid Interface Sci. 2005, 288, 301-303.

(21) Sun, X.; Dong, S.; Wang, E. One-Step Polyelectrolyte-Based Route to Well-Dispersed Gold Nanoparticles: Synthesis and Insight. Mater. Chem. Phys. 2006, 96, 29-33.

(22) Mikami, T.; Takayasu, Y.; Hirasawa, I. PEI-Assisted Preparation of $\mathrm{Au}$ Nanoparticles via Reductive Crystallization Process. Chem. Eng. Res. Des. 2010, 88, 1248-1251.

(23) Kretschmer, F.; Mansfeld, U.; Hoeppener, S.; Hager, M. D.; Schubert, U. S. Tunable Synthesis Of Poly(Ethylene Imine)-Gold Nanoparticle Clusters. Chem. Commun. 2014, 50, 88-90.

(24) Zhou, B.; Zheng, L.; Peng, C.; Li, D.; Li, J.; Wen, S.; Shen, M.; Zhang, G.; Shi, X. Synthesis and Characterization of PEGylated Polyethylenimine-Entrapped Gold Nanoparticles for Blood Pool and Tumor CT Imaging. ACS Appl. Mater. Interfaces 2014, 6, 17190-17199.

(25) Li, A.; Zhou, B.; Alves, C. S.; Xu, B.; Guo, R.; Shi, X.; Cao, X. Mechanistic Studies of Enhanced PCR Using PEGylated PEI-Entrapped Gold Nanoparticles. ACS Appl. Mater. Interfaces 2016, 8, 25808-25817.

(26) Chen, C.-C.; Hsu, C.-H.; Kuo, P.-L. Effects of Alkylated Polyethylenimines on the Formation of Gold Nanoplates. Langmuir 2007, 23, 6801-6806.

(27) Köth, A.; Appelhans, D.; Prietzel, C.; Koetz, J. Asymmetric Gold Nanoparticles Synthesized in the Presence of Maltose-Modified Poly(Ethyleneimine). Colloids Surf., A 2012, 414, 50-56.

(28) Mészáros, R.; Thompson, L.; Bos, M.; Varga, I.; Gilányi, T. Interaction of Sodium Dodecyl Sulfate with Polyethyleneimine: Surfactant-Induced Polymer Solution Colloid Dispersion Transition. Langmuir 2003, 19, 609-615. 
(29) Mezei, A.; Mészáros, R.; Varga, I.; Gilányi, T. Effect of Mixing on the Formation of Complexes of Hyperbranched Cationic Polyelectrolytes and Anionic Surfactants. Langmuir 2007, 23, 4237-4247.

(30) Mezei, A.; Pojják, K.; Mészáros, R. Nonequilibrium Features of the Association between Poly(vinylamine) and Sodium Dodecyl Sulfate: The Validity of the Colloid Dispersion Concept. J. Phys. Chem. B 2008, 112, 9693-9699.

(31) Štěpánek, M.; Hajduová, J.; Procházka, K.; Šlouf, M.; Nebesářová, J.; Mountrichas, G.; Mantzaridis, C.; Pispas, S. Association of Poly(4hydroxystyrene)-block-Poly(Ethylene oxide) in Aqueous Solutions: Block Copolymer Nanoparticles with Intermixed Blocks. Langmuir 2012, 28, 307-313.

(32) Campbell, R. A.; Arteta, M. Y.; Angus-Smyth, A.; Nylander, T.; Noskov, B. A.; Varga, I. Direct Impact of Nonequilibrium Aggregates on the Structure and Morphology of Pdadmac/SDS Layers at the Air/ Water Interface. Langmuir 2014, 30, 8664-8674.

(33) Fegyver, E.; Mészáros, R. Complexation between Sodium Poly(styrenesulfonate) and Alkyltrimethylammonium bromides in the Presence of Dodecyl maltoside. J. Phys. Chem. B 2015, 119, 5336-5346.

(34) Bodnár, K.; Fegyver, E.; Nagy, M.; Mészáros, R. Impact of Polyelectrolyte Chemistry on the Thermodynamic Stability of Oppositely Charged Macromolecule/Surfactant Mixtures. Langmuir 2016, 32, 1259-1268.

(35) Ferreira, G. A.; Piculell, L.; Loh, W. Addition of n-Alcohols Induces a Variety of Liquid-Crystalline Structures in Surfactant-Rich Cores of Dispersed Block Copolymer/Surfactant Nanoparticles. ACS Omega 2016, 1, 1104-1113.

(36) Dedinaite, A.; Mészáros, R.; Claesson, P. M. Effect of Sodium Dodecyl Sulfate on Adsorbed Layers of Branched Polyethylene Imine. J. Phys. Chem. B 2004, 108, 11645-11653.

(37) Varga, I.; Mezei, A.; Mészáros, R.; Claesson, P. M. Controlling the Interaction of Poly(Ethylene Imine) Adsorption Layers with Oppositely Charged Surfactant by Tuning the Structure of the Preadsorbed Polyelectrolyte Layer. Soft Matter 2011, 7, 10701-10712.

(38) Xia, H.; Xiahou, Y.; Zhang, P.; Ding, W.; Wang, D. Revitalizing the Frens Method To Synthesize Uniform, Quasi- Spherical Gold Nanoparticles with Deliberately Regulated Sizes from 2 to $330 \mathrm{~nm}$. Langmuir 2016, 32, 5870-5880.

(39) Wang, S. T.; Yan, J. C.; Chen, L. Formation of gold nanoparticles and self-assembly into dimer and trimer aggregates. Mater. Lett. 2005, 59, 1383-1386.

(40) Mezei, A.; Mészáros, R. Novel Method for the Estimation of the Binding Isotherms of Ionic Surfactants on Oppositely Charged Polyelectrolytes. Langmuir 2006, 22, 7148-7151. 